\title{
Probability learning in immature and adult rats'
}

NICHOLAS LONGO AND LARRY R. BEIDEMAN

COLGATE UNIVERSITY

Adult and immature rats were studied in two probability learning tasks. In a black-white Y-maze problem both groups maximized, choosing the majority stimulus about $95 \%$ of the time. In a brightness discrimination employing automated procedures, however, both groups matched the reward ratios but matching was found to be partly a function of systematic patterns of response and partly an artifact of averaging.

Phyletic comparisons of probability learning have been described for a wide variety of animals (Bitterman, 1965). In general, these studies have shown that while rats and monkeys "maximize" in two-choice learning situations, lower species such as pigeons, fish and cockroaches tend to show a correspondence of choice ratio and reward ratio. What importance is to be attributed to this mammalian-submammalian dichotomy? One hypothesis offered by Gonzalez et al (1964) is in terms of the higher cortical development of mammals. Bilaterally decorticated rats in these studies perform in much the same way as lower species in probability learning tasks and also fail to show the characteristic progressive improvement in habit reversals.

The present experiments attempt to study probability learning as a function of ontogenetic changes in the rat brain. The size of the rat cerebral cortex increases in a negatively accelerated manner from birth to about 120 days, after which there is little further growth (Zeman \& Innes, 1963). In the experiments below, adult and immature rats were studied in conventional $\mathrm{Y}$-mazes and in automated experimental chambers.

Method

The adult Ss were 24 albino rats of the SpragueDawley strain, 90-120 days of age, and the immature Ss were 24 rats of the same strain but only 21 days of age at the beginning of the experiment. Half of each age group was run in automated experimental chambers in which the two discriminanda were projected on push-panels, and half were run on a black-white discrimination in elevated $\mathrm{Y}$-mazes. In the first experiment, the two push-panels were located at one end of a Plexiglas chamber and a standard rat lever at the other end. Two sizes of chambers were used to accommodate the two different age Ss. All Ss were trained to depress the back lever which in turn started the trial by illuminating the two stimuli at the front of the chamber. An opaque vertical partition midway between the front panels and the back lever forced the Ss to look at the two projected stimuli before making a choice.

The Ss were first trained on a 0:100 problem in what was essentially a brightness discrimination (a 1-in. circular patch of light vs, a 1-in. open circle). Half of the Ss were consistently rewarded for one stimulus and half for the other. The stimuli appeared left or right on a random schedule. A correct response delivered a 45-mg food pellet which was followed by a 6-sec. interval of darkness before the back lever was illuminated and again activated to turn on the two front stimuli. After an incorrect response, both stimuli were turned off and after a 6 sec. period of darkness, the back lever was illuminated to indicate that it was available again to turn on the two stimuli. After four consecutive errors, a "guidance" procedure was used where only the correct stimulus was presented. When each $S$ reached a criterion of $18 / 20$ correct, it was switched to a 70:30 problem where the formerly incorrect stimulus of the $0: 100$ problem became the majority stimulus. Two 20-trial sessions were given daily. Reinforcement to the minority stimulus was randomly distributed within the 20 trials with the restriction that it occurred at least once in each block of five trials. All experimental contingencies and recording of data were controlled by automatic programming apparatus. A 23-hr. deprivation schedule was used for all Ss.

In the second experiment two elevated $\mathrm{Y}$-mazes were used, one scaled down for the young Ss. Each arm of the maze led to an opening which was blocked by a black or white card. The correct card could be knocked over admitting the animal to the goal compartment while the incorrect card was locked in place. The mazes were painted a neutral gray. As in the first experiment, all Ss were first trained on a 0:100 problem and after reaching a criterion of $18 / 20$ correct, they were switched to a 70:30 problem using the previously described procedures. If the $S$ made an incorrect choice it was returned to the starting point until it made a correct response or made three consecutive repetitive errors following which the experimenter blocked the incorrect path and guided the $S$ to the correct card. Reward consisted of access to wet mash for 10-sec.

\section{Results and Discussion}

Figure 1 shows the results obtained with the automated chamber in terms of percent choice to the majority stimulus. Both the immature and adult Ss rapidly reach criterion in the 0:100 discrimination. When switched to the 70:30 problem, both groups approach and remain at the $70 \%$ choicelevel. The training of the immature Ss was continued for an additional 900 trials (as indicated by the last 200 trials in Fig. 1) but the choice level did not change substantially from the earlier performance.

This apparent matching differs in several respects from the random matching for fish and pigeons (Bitterman, 1965). Daily performance for both groups showed 


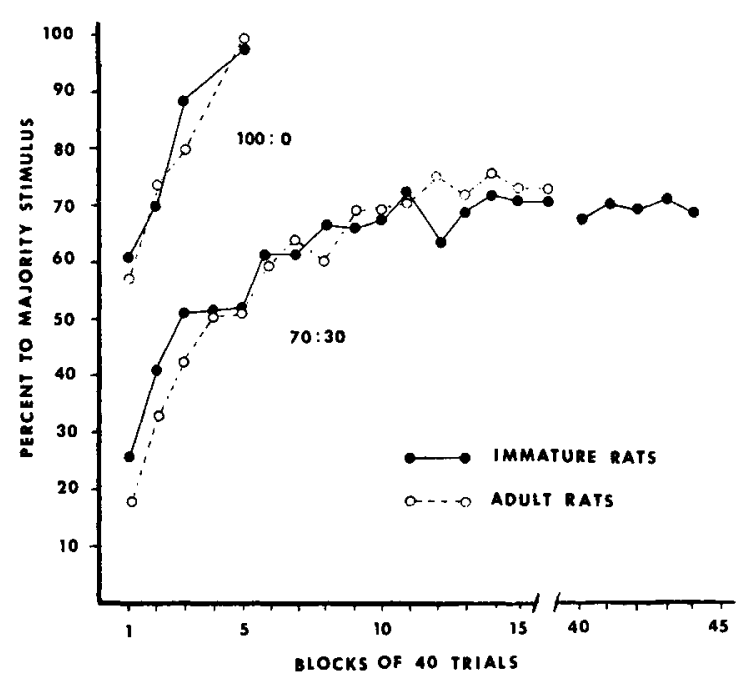

Fig. 1. Probability learning in immature and adult rats with the automated chamber.

considerable variability, ranging from 50 to $90 \%$ choice level, so that to some extent the matching here is an artifact of averaging. In the three or four Ss in each age group where a consistent matching level was observed, it was not the random matching described by Bitterman (1965). For example, the percent choice of the majority stimulus after a minority reinforcement was $24 \%$, while the choice after a majority reinforcement was $92 \%$. Thus, matching here is a function of systematic patterns of choice very similar to the data reported by Hickson (1961).

In contrast to the above results, Fig. 2 shows that in the Y-maze, both groups of rats definitely maximize. Both the adult and immature Ss rapidly learn the 0:100 black-white problem and reach the 95\% level when switched to the 70:30 problem. Immature Ss as young as 35-40 days of age maximize as readily as adult rats. It is unfortunate that practical methodological considerations do not permit comparisons at earlier stages of development.

Both types of experimental procedures, therefore, do not reveal any essential differences between the performance of adult and immature rats. In the Y-maze, both age groups maximize, and in the automated chamber matching for both groups is partly a function of sequential dependency and partly an artifact of averaging.

One can only speculate about the discrepancy in results between the two experiments. ${ }^{2}$ our purpose in

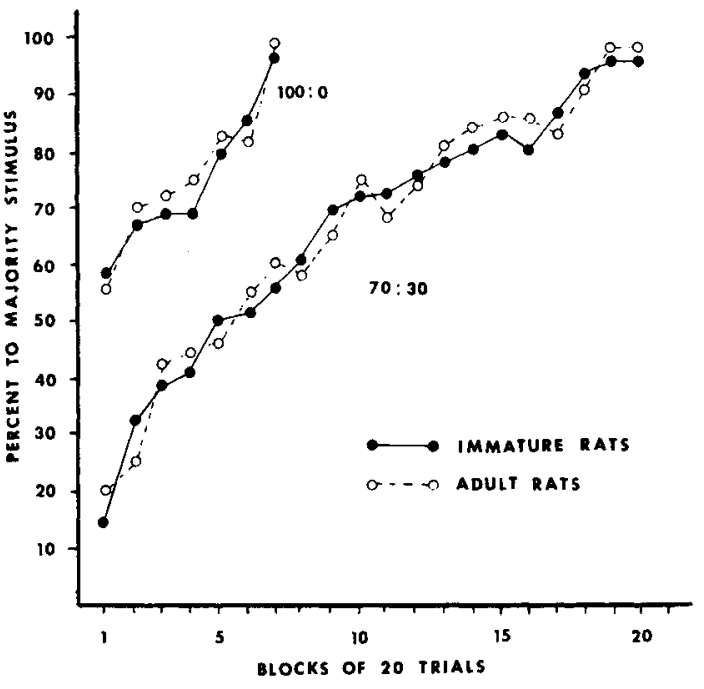

Fig. 2. Probability learning in immature and adult rats with the Y-maze.

studying probability learning in the automated chamber was to make the experimental procedures similar to the fish and pigeon studies and to make the procedures efficient and objective. Although essentially analogous, the $\mathrm{Y}-\mathrm{maze}$ and the automated procedures differ in many respects, but which of these differences account for the dissimilar performance is difficult to assess. For example, handling of rats after an incorrectchoice is conceivably more aversive than the brief period of darkness in the chamber. These studies again emphasize the difficulty in making broad generalizations on the basis of isolated experimental data.

\section{References}

Bitterman, M. E. Phyletic differences in learning. Amer. Psychologist, $1965,20,396-410$.

Gonzalez, R. C., Roberts, W. A., \& Bitterman, M. E. Learning in adult rats with extensive cortical lesions made in infancy. Amer. J. Psychol., 1964, 77, 547-562.

Hickson, R. H. Response probability in a two choice learning situation with varying probability of reinforcement. $J$, exp. Psychol., $1961,62,138-144$.

Zemen, W., \& Innes, J. R. M. Craigie's Neuroanatomy of the Rat, New York: Academic Press, 1963.

\section{Notes}

1. Supported by Public Health Service Grant MH 108-46 and a grant from Wallace Laboratories.

2. Similar experiments with the automated chamber in which we used a variety of other stimuli, correction procedures, and longer periods of darkness after an error yielded essentially the same results. 